\title{
Nutrition support to patients undergoing gastrointestinal surgery
} Nicola Ward*

\author{
Address: Department of Pharmacy, Glenfield Hospital, University Hospitals of Leicester, NHS Trust, Leicester, UK \\ Email: Nicola Ward* - nicola.ward@uhl-tr.nhs.uk \\ * Corresponding author
}

Published: 0 I December 2003

Nutrition Journal 2003, 2:18

This article is available from: http://www.nutritionj.com/content/2/I//8

(c) 2003 Ward; licensee BioMed Central Ltd. This is an Open Access article: verbatim copying and redistribution of this article are permitted in all media for any purpose, provided this notice is preserved along with the article's original URL.

\begin{abstract}
Nutritional depletion has been demonstrated to be a major determinant of the development of post-operative complications. Gastrointestinal surgery patients are at risk of nutritional depletion from inadequate nutritional intake, surgical stress and the subsequent increase in metabolic rate.

Fears of postoperative ileus and the integrity of the newly constructed anastomosis have led to treatment typically entailing starvation with administration of intravenous fluids until the passage of flatus. However, it has since been shown that prompt postoperative enteral feeding is both effective and well tolerated. Enteral feeding is also associated with specific clinical benefits such as reduced incidence of postoperative infectious complications and an improved wound healing response. Further research is required to determine whether enteral nutrition is also associated with modulation of gut function.

Studies have indicated that significant reductions in morbidity and mortality associated with perioperative Total Parenteral Nutrition (TPN) are limited to severely malnourished patients with gastrointestinal malignancy. Meta-analyses have shown that enteral nutrition is associated with fewer septic complications compared with parenteral feeding, reduced costs and a shorter hospital stay, so should be the preferred option whenever possible.

Evidence to support pre-operative nutrition support is limited, but suggests that if malnourished individuals are adequately fed for at least 7-10 days preoperatively then surgical outcome can be improved.

Ongoing research continues to explore the potential benefits of the action of glutamine on the gut and immune system for gastrointestinal surgery patients. To date it has been demonstrated that glutamine-enriched parenteral nutrition results in reduced length of stay and reduced costs in elective abdominal surgery patients. Further research is required to determine whether the routine supplementation of glutamine is warranted.

A limitation for targeted nutritional support is the lack of a standardised, validated definition of nutritional depletion. This would enable nutrition support to be more readily targeted to those surgical patients most likely to derive significant clinical benefit in terms of improved post-operative outcome.
\end{abstract}

\section{Introduction}

Protein-energy malnutrition is a common problem in hospital patients. Studies have reported $40 \%$ of surgical and medical patients to be malnourished on admission to 
hospital. The majority of patients experienced nutritional depletion during the course of their hospital admission, which was more severe in those patients who were already depleted at the time of their admission [1]. The consequences of pre-operative malnutrition were first recognised in the 1930's. Studley observed a direct relationship between preoperative weight loss and operative mortality rate, independent of factors such as age, impaired cardiorespiratory function and type of surgery [2]. The importance of nutritional depletion as a major determinant of the development of postoperative complications has subsequently been confirmed by Giner et al [3]. The absence of a standardised definition of nutritional depletion has led to surrogate markers of nutritional status being utilised. Albumin, muscle function tests, immunological status and weight loss are used as these show correlation with postoperative morbidity and mortality.

Nutritional depletion is associated with changes in body composition, tissue wasting and impaired organ function which leads to impaired immune and muscle function. Thus, depleted patients are at risk from infectious complications and cardiorespiratory impairment $[4,5]$. Patients who undergo gastrointestinal surgery are at risk of nutritional depletion from inadequate nutritional intake; both preoperatively and postoperatively, the stress of surgery and the subsequent increase in metabolic rate.

More recently, ensuring adequate nutritional intake has been a major focus of perioperative care and research has focused on the methods of delivering nutritional support, their comparative clinical benefits and minimising the metabolic changes associated with surgical trauma.

\section{Metabolic changes in surgical patients}

The physiological stress of surgical trauma causes a surge of sympathetic activity and an associated rise in catecholamine secretion. These changes are transient. A more prolonged hypermetabolic state associated with a pronounced negative nitrogen balance then follows. Metabolic rate is typically increased by about $10 \%$ postoperatively [6]. If adequate nutritional support is not provided at this stage then excessive skeletal muscle proteolysis occurs with further depression of metabolism. Increased energy expenditure is associated with a range of hormonal responses that occur as a result of surgical trauma. Cytokines, including Tumour Necrosis Factor (TNF) and interleukins (IL-1 and IL-6) have an important role in determining longer-term metabolic changes [7]. These changes may not be clinically relevant unless postoperative sepsis or trauma follows surgery but in conjunction with preoperative starvation often results in a significant negative nitrogen balance.

\section{Physiological changes in surgical patients}

It has been proved that intestinal permeability is increased two to fourfold in the immediate postoperative period, although this normalises within five days [8]. In addition, nutritional depletion is associated with increased intestinal permeability and a decrease in villous height [9]. These findings have lead to the investigation of treatments aimed at maintaining an intact mucosal barrier. Increased intestinal permeability indicates a failure of the gut barrier function to exclude endogenous bacteria and toxins. These have been proposed as causative agents in the systemic inflammatory response syndrome, sepsis and multiorgan failure. However, there has been a failure thus far to prove a correlation between failure of gut barrier function and septic complications after major upper gastrointestinal failure [10].

\section{Clinical benefits to surgical patients}

Nutritional support leads to improved nutritional status and clinical outcome in severely depleted patients [11]. Studies of postoperative nutritional support have demonstrated reduced morbidity and reduced length of hospital stay [12]. There is also evidence that artificial nutritional support in malnourished patients is cost effective by reducing the costs associated with length of stay and morbidity and improved quality of life [13]. It is important, however, to consider the most clinically appropriate and beneficial means of delivering nutritional support to surgical patients.

\section{Enteral nutrition}

Conventional treatment after bowel resection has typically entailed starvation with administration of intravenous fluids until passage of flatus, principally due to concerns over post-operative ileus. This was based on the assumption that oral feeding may not be tolerated in the presence of ileus and the integrity of the newly constructed anastomosis may be compromised. However, small intestinal motility recovers $6-8$ hours after surgical trauma and moderate absorptive capacity exists even in the absence of normal peristalsis [14]. It has since been shown that postoperative enteral feeding in patients undergoing gastrointestinal resection is safe and well tolerated even when started within 12 hours of surgery $[15,16]$. The commonest observed adverse effects were gastrointestinal, such as abdominal cramps and bloating [16].

An appropriate delivery method should be selected, depending on the anticipated duration of enteral feeding, aspiration risk and gastrointestinal anatomy. No specific clinical or nutritional advantages have been shown for jejunostomy feeding and this route should be reserved for patients in whom naso-gastric or naso-jejunal feeding is not feasible or safe [17]. 
Enteral feeding has been shown to result in some specific clinical benefits, including reducing the incidence of postoperative infectious complications [11] and an improved wound healing response [18]. Enteral nutrition may have other beneficial effects including altering antigen exposure and influencing oxygenation of the gut mucosa. More research is required in this area to elucidate whether enteral nutrition truly modulates gut function or whether tolerance of enteral nutrition is predominantly indicative of a patient with healthy organ function [19].

\section{Parenteral nutrition}

A large multi-centre clinical trial has shown no significant reduction in morbidity or mortality when Total Parenteral Nutrition (TPN) was administered perioperatively to a heterogeneous group of surgical patients. Stratification of patients in this trial according to nutritional status showed that patients with mild malnutrition did not benefit from TPN but had more infectious complications. This led the authors to conclude that perioperative TPN should be limited to severely malnourished patients in the absence of other specific indications [20]. Subsequent studies have principally focussed on severely malnourished patients with gastrointestinal malignancy. These patients have been shown to experience clinically significant reductions in both infectious and non-infectious complications when fed parenterally for a minimum of ten days pre-operatively [21]. A recent meta-analysis of 27 randomised controlled trials concluded that TPN has no statistically significant effects overall on mortality or morbidity in surgical patients. The most recent studies analysed were of better methodological quality and showed fewer benefits than earlier studies. Studies which included only malnourished patients demonstrated a trend to a reduction in complication rates [22].

\section{Enteral versus parenteral nutrition}

Each route of delivery of nutritional support is associated with different complications. Generally, the complications associated with parenteral nutrition are associated with greater morbidity than those associated with enteral nutrition due to the invasive nature of administration. The route of administration also has effects on organ function, particularly the intestinal tract. Substrates delivered by the enteral route are better utilised by the gut than those administered parenterally. In addition, enteral feeding when compared with TPN solutions may prevent gastrointestinal mucosal atrophy, attenuate the trauma stress response, maintain immunocompetence and preserve normal gut flora [23].

A meta-analysis comparing the nutritional efficacy of early enteral and parenteral nutrition in high-risk surgical patients found that early postoperative enteral nutrition was effective and associated with reduced septic morbidity rates compared with those administered TPN even when catheter sepsis was excluded from the analysis [23]. Enteral nutrition is also an effective option in severely malnourished patients with gastrointestinal cancer and is associated with fewer complications, a shorter post-operative hospital stay [25] and reduced costs compared with TPN [26]. The principal conclusions from these studies was that the enteral route should be used whenever possible, but if the enteral route will not be available for more than one week early administration of TPN should be considered.

\section{Preoperative versus post operative}

Evidence to support preoperative nutrition support is limited but suggests that if malnourished individuals are adequately fed for at least 7-10 days preoperatively then surgical outcome can be improved [20]. The obvious disadvantage of this is the increased length of hospital stay resulting from admission for nutritional support and the delay in surgical intervention. There is also some evidence to support preoperative nutrition support in patients with inflammatory bowel disease [27]. Studies have also been carried out which cast doubt on the benefits of the standard preoperative fast and have shown reductions in the postoperative catabolic response and improved wellbeing [28]. As discussed earlier, there is substantially more evidence to support early post-operative nutritional intervention by an appropriate route.

\section{Immunonutrition}

In addition to ongoing research ascertaining the specific benefits of the routes of delivery for nutrition support, more recent research has also focussed on the composition of nutritional regimens. In particular, much attention has been paid to the potential for specific nutrients to influence the metabolic response to disease.

Glutamine is the most abundant free amino acid in the extra and intracellular compartments. It plays a vital role in nitrogen transport and acid base homeostasis and is a fuel for rapidly dividing cells such as enterocytes, lymphocytes and fibroblasts. It is also involved in antioxidant defence mechanisms by influencing glutathione synthesis. In situations of severe stress or nutritional depletion the demand for glutamine may exceed the body's capacity to synthesise it. Studies have explored the benefits of glutamine-enriched parenteral nutrition regimens, particularly on the gut and immune system. It has been demonstrated that the addition of glutamine to parenteral nutrition regimens given to patients after elective abdominal surgery results in a reduced length of hospital stay [29] and reduced costs [30]. This was accompanied by an improved nitrogen balance and quicker lymphocyte recovery [29]. Glutamine has also been shown to maintain intestinal permeability in postoperative patients [31]. 
Studies regarding the role of two other potential immunonutrients, arginine and n-3 fatty acids, in gastrointestinal surgery patients have not yet been published.

\section{Conclusions}

Randomised controlled trials provide evidence to support the use of enteral feeding in surgical patients and indicate no increased morbidity or mortality. However, no metaanalyses have been carried out to pool the data from a plethora of mainly small trials. To date, these show no reductions in mortality have been shown from enterally fed surgical patients. Some useful meta-analyses have been published for parenterally feeding surgical patients, although many studies were small and of flawed methodological quality as they did not take into account the many surgical and anaesthetic variables that can influence post-operative outcomes. Comparative studies show that, compared with parenteral nutrition, enteral nutrition is well tolerated and is associated with reduced septic morbidity, costs and length of stay. These studies form the basis of the current practice of the enteral feeding route being used wherever possible. Further research is needed to elucidate whether, alongside improvements in surgical technique and perioperative care, enteral nutrition will be associated with overall reductions in morbidity and mortality.

Further research is required to clearly identify which surgical patients will significantly benefit from specific nutritional intervention. This is problematic as assessment of nutritional status is not straightforward and there is also an absence of a standardised definition of nutritional depletion. A standardised, validated definition of nutritional depletion would enable nutrition support to be targeted to those surgical patients most likely to derive significant clinical benefit in terms of improved postoperative outcome. This would also facilitate direct comparison of trial data for large meta-analyses involving "malnourished" patients to provide robust, evidence based guidelines for nutritional support of surgical patients.

\section{Competing interests}

The author has no competing interests.

\section{Authors' contributions}

NW carried out the literature search, reviewed the papers and prepared the manuscript.

\section{Acknowledgements}

The author kindly acknowledges the assistance of Mr ADN Scott and Dr David Gerrett in reviewing and commenting on the manuscript.

\section{References}

I. McWhirter JP, Pennington CR: The incidence and recognition of malnutrition in hospital. BMJ I994, 308:945-8.
2. Studley HO: Percentage weight loss, a basic indicator of surgical risk in patients with chronic peptic ulcer. JAMA 1936, 106:458-460.

3. Giner M, Laviano A, Meguid MM, Gleason JR: In I 995 a correlation between malnutrition and poor outcome in critically ill patients still exists. Nutrition 1996, I 2:23-9.

4. Windsor JA, Hill GL: Risk factors for post operative pneumonia: the importance of protein depletion. Ann Surg 1988, 17:181-5.

5. Arora NS, Rochester DF: Respiratory muscle strength and maximal voluntary ventilation in undernourished patients. Am Rev Respir Dis 1982, I 26:5-8.

6. Kinney JM, Duke JH Jr, Long CL, Gump FE: Tissue fuel and weight loss after injury. J Clin Path 1970, Suppl 4:65-72.

7. Douglas RG, Shaw JHF: Metabolic response to sepsis and trauma. Br J Surg 1989, 76: II5-122.

8. Beattie AH, Prach AT, Baxter AT, Pennington CR: A randomised controlled trial evaluating the use of enteral nutritional supplements postoperatively in malnourished surgical patients. Gut 2000, 46:8I3-8I8.

9. van der Hulst RR, von Meyenfeldt MF, van Freel BK, Thunnissen FB, Brummer RJ, Arends JW, Soeters PB: Gut permeability, intestinal morphology, and nutritional depletion. Nutrition I998, I 4: I-6.

10. Kanwar S, Windsor AC, Welsh F, Barclay GR, Guillou PJ, Reynolds JV: Lack of correlation between failure of gut barrier function and septic complications after major upper gastrointestinal surgery. Ann Surg 2000, 23I:88-95.

II. Beier-Holgersen SR, Boesby S: Influence of postoperative enteral nutrition on post surgical infections. Gut 1996, 39:833-5.

12. Askanazi J, Starker PN, Olsson C, Hensle TW, Lockhart SH, Kinney JM, Lasala PA: Effect of immediate post-operative nutritional support on the length of hospitalisation. Ann Surg 1986, 203:236-9.

13. Robinson G, Goldstein M, Levine G: Impact of nutritional status on DRG length of stay. JPEN I 987, I I:49-5I.

14. Woods JH, Erickson LW, Condon RE: Post-operative ileus: a colonic problem. Surgery 1978, 84:527-533.

15. Reissman P, Teoh TA, Cohen SM, Weiss EG, Nogueras JJ, Wexner SD: Is early oral feeding safe after elective colorectal surgery? A prospective randomized trial. Ann Surg 1995, 222:73-77.

16. Braga M, Gianotti L, Gentilini S, Liotta S, Di Carlo V: Feeding the gut early after digestive surgery: results of a nine-year experience. Clinical Nutrition 2002, 21:59-65.

17. Smith RC, Hartemink RJ, Hollinshead JW, Gillett DJ: Fine bore jejunostomy feeding following major abdominal surgery: a controlled randomised clinical trial. Br J Surg 1985, 72:458-61.

18. Schroeder D, Gillanders L, Mahr K, Hill GL: Effects of immediate postoperative enteral nutrition on body composition, muscle function and wound healing. JPEN I991, 15:376-383.

19. Reynolds JV: Gut barrier function in the surgical patient. $\mathrm{Br} J$ Surg 1996, 83:1668-1669.

20. Veterans Affairs Total Parenteral Nutrition Cooperative Study Group: Periperative Total Parenteral Nutrition in surgical patients. NEJM 199I, 325:525-32.

21. Bozzetti F, Gavazzi C, Miceli R, Rossi N: Perioperative total parenteral nutrition in malnourished, gastrointestinal cancer patients: a randomised, clinical trial. JPEN 2000, 24:7-I4.

22. Heyland DK, Montalvo M, MacDonald S, Keefe L, Xiang YS, Drover JW: Total Parenteral Nutrition in the surgical patient: a meta-analysis. Can J Surg 200I, 44: I02-II.

23. Saito $H$, Trocki $O$, Alexander JW: The effect of route of nutrient administration on the nutritional state, catabolic hormone secretion, and gut mucosal integrity after burn injury. JPEN 1987, II:I-7.

24. Moore FA, Feliciano DV, Andrassy RJ, McArdle AH, Booth FV, Morgenstein-Wagner TB, Kellum JM, Welling RE, Moore EE: Early enteral feeding, compared with parenteral, reduced postoperative septic complications. The results of a meta-analysis. Ann Surg 1992, 216:172-183.

25. Bozzetti F, Braga M, Gianotti L, Gavazzi C, Mariani L: Postoperative enteral versus parenteral nutrition in malnourished patients with gastrointestinal cancer: a randomised multicentre trial. Lancet 200I, 358: 1487-92.

26. Braga M, Gianotti L, Gentilini O, Parisi V, Salis C, Di Carlo V: Early postoperative enteral nutrition improves gut oxygenation 
and reduces costs compared with total parenteral nutrition. Crit Care Med 200I, 29:242-248.

27. Rombeau JL, Barot LR, Williamson CE, Mullen JL: Preoperative total parenteral nutrition and surgical outcome in patients with inflammatory bowel disease. Am J Surg 1982, 1 43: 1 $39-43$.

28. Ljungqvist O, Soreide E: Preoperative fasting. Br J Surg 2003, 90:400-6.

29. Morlion BJ, Stehle P, Wachtler P, Siedhoff HP, Koller M, Konig W, Furst $P$, Puchstein $C$ : Total parenteral nutrition with glutamine dipeptide after major abdominal surgery. Ann Surg 1998, 227:302-8.

30. Mertes N, Schulzki C, Goeters C, Winde G, Benzing S, Kuhn KS, van Aken H, Stehle P, Furst P: Cost containment through L-alanylL-glutamine supplemented total parenteral nutrition after major abdominal surgery: a prospective randomised doubleblind controlled study. Clin Nutrition 2000, 19:395-40I.

31. Jiang ZM, Cao JD, Zhu XG, Zhao WX, Yu JC, Ma EL, Wang XR, Zhu MW, Shu H, Liu YW: The impact of alanyl-glutamine on clinical safety, nitrogen balance, intestinal permeability, and clinical outcome in postoperative patients: a randomised, doubleblind, controlled study of I 20 patients. JPEN I999, 23:S62-66.

Publish with Bio Med Central and every scientist can read your work free of charge

"BioMed Central will be the most significant development for disseminating the results of biomedical research in our lifetime. "

Sir Paul Nurse, Cancer Research UK

Your research papers will be:

- available free of charge to the entire biomedical community

- peer reviewed and published immediately upon acceptance

- cited in PubMed and archived on PubMed Central

- yours - you keep the copyright

Submit your manuscript here:

http://www.biomedcentral.com/info/publishing_adv.asp 